\title{
Asymmetric reheating from a symmetric inflationary potential
}

\author{
James M. Cline* and Jean-Samuel Roux ${ }^{\dagger}$ \\ McGill University, Department of Physics, 3600 University St., Montréal, QC H3A2T8 Canada
}

\begin{abstract}
We explore a model of two-field inflation with nonminimal kinetic terms in which two identical matter sectors decoupled from each other may reheat to different temperatures while preserving the symmetry of the Lagrangian. This scenario is motivated by mirror dark matter models in which the temperature of the mirror sector is constrained to be $T^{\prime} \lesssim 0.5 T$ by big bang nucleosynthesis and the cosmic microwave background. For a given class of nonminimal kinematic terms, we find that the symmetric field trajectory $X=Y$ is a repeller solution, such that any randomly-occurring asymmetry in the initial conditions is amplified by many orders of magnitude during inflation, far beyond what canonical power-law models can achieve. Isocurvature fluctuations are strongly suppressed in this model, but a $\mathcal{O}(0.03-0.07)$ tensor-to-scalar ratio could be observed in the near future. The range of potential parameters compatible with Planck constraints is shown to be much larger than in corresponding single-field models. This occurs through a mechanism for lowering the spectral index that we dub CTHC: curved trajectory at horizon crossing.
\end{abstract}

\section{INTRODUCTION}

Mirror models were perhaps the original paradigm for a hidden sector in particle physics [1-3]. A copy of the standard model (SM) field content and gauge group is hypothesized, which in its simplest form is exact, so that the two sectors are related by a discrete $Z_{2}$ mirror symmetry. This form is subject to significant cosmological constraints on the additional relativistic degrees of freedom - mirror photons $\gamma^{\prime}$ and neutrinos $\nu^{\prime}$ - that would increase the Hubble expansion rate at early times, in contradiction to the successful predictions of big bang nucleosynthesis (BBN) and the cosmic microwave background (CMB). To circumvent the bound on the effective number of neutrino species $N_{\text {eff }}$, one must either break the mirror symmetry by making $\gamma^{\prime}$ and $\nu^{\prime}$ massive, or in the case of exact $Z_{2}$ symmetry, arrange for the initial conditions of the Universe to create a lower temperature $T^{\prime}$ in the mirror sector than in the visible one, with $x \equiv T^{\prime} / T \lesssim 0.5$ [4, 5].

Similar frameworks like Twin Higgs models (see [6] for a recent example) or parity solutions to the strong $\mathrm{CP}$ problem [7] break the mirror symmetry at late times. However, if at least one mirror species like $\gamma^{\prime}$ remains massless, those scenarios also require $x \lesssim 0.6$ to satisfy cosmological bounds on $N_{\text {eff }}$. The breaking of the symmetry might facilitate entropy transfers that cool the mirror sector, but it is also possible that mirror and visible species decouple when the symmetry still holds, in which case the temperature hierarchy must originate from early universe dynamics, when the Lagrangian was still symmetric.

One may wonder how likely it is to realize perfect mirror symmetry in a complete model including inflation, such that the relative temperatures in the two sectors differ as required by the constraints. In Refs. 4, 8, 9, it

\footnotetext{
* jcline@physics.mcgill.ca

$\dagger$ jsroux@hep.physics.mcgill.ca
}

was noted that asymmetric reheating would generically occur in models with two inflatons, one for each sector, due to differences in the initial conditions. Here we revisit this idea, in the light of current $\mathrm{CMB}$ constraints from Planck [10] 1 ]

We consider two-field chaotic inflation with decoupled potentials of the form

$$
V_{\text {tot }}=V(X)+V(Y)=\lambda \frac{|X|^{p}}{m_{P}^{p-4}}+\lambda \frac{|Y|^{p}}{m_{P}^{p-4}},
$$

(where $m_{P}$ is the reduced Planck mass) plus respective couplings of each field to its own sector's matter particles, to accomplish reheating. In the case of a purely quadratic potential $(p=2)$ the solutions are such that the ratio of the two inflatons $Y / X$ remains constant during inflation 9. Then the ratio of the reheating temperatures goes as $T^{\prime} / T=\left(Y^{2} / X^{2}\right)^{1 / 4}$, and is thereby analytically determined in terms of the initial conditions. This example is now ruled out by Planck data [10, which strongly disfavors chaotic inflation models that have convex potentials.

In the following, we study a model with noncanonical inflaton kinetic terms, proposed in Ref. [5], that generates a temperature hierarchy by the spontaneous breaking of mirror symmetry by the initial values of the inflatons. At large field values, it is equivalent to Eq. (1) with fractional values of $p$, and we therefore consider both kinds of models. Such fractional power-law potentials have been proposed in the context of string theory 12 19 or supergravity [20]. We will show, somewhat surprisingly,

\footnotetext{
${ }^{1}$ An early proposal for getting asymmetric reheating was given in ref. [1], which proposed a 'double-bubble inflation' model where the ordinary and mirror inflatons finish inflation by bubble nucleation at different (random) times. In this case the first sector to undergo reheating gets exponentially redshifted until the second field nucleates a bubble of true vacuum. However this is in the context of "old inflation" driven by false vacua, which is untenable because the phase transitions never complete.
} 
that the initial values $X_{i}, Y_{i}$ cannot be too different from each other, while remaining consistent with Planck constraints on the CMB observables; nevertheless, a small initial asymmetry is typically amplified during inflation into a very large asymmetry in the final temperature ratio. We will also show that the two-field inflationary scenarios lead to much better agreement with the CMB than their single-field counterparts, due to the effect of curvature in the inflaton trajectory in the $X-Y$ field space, at the time of horizon crossing. We abbreviate this effect by "CTHC." It was previously observed in the context of multifield inflation with fractional power law potentials in Ref. [21, with emphasis on many inflatons having random potential parameters.

In Section II] we introduce the noncanonical two-field models, and the corresponding canonical models that are equivalent at large field values. The numerical techniques used are described there, along with analytical approximations that explain the qualitative behavior of the exact solutions. We illustrate our results for three benchmark parameter choices in Section III. This is followed in Section [V] by a description of a Monte Carlo search of the full parameter space for models that are consistent with all constraints. We give conclusions in Section $\mathrm{V}$.

\section{NONCANONICAL TWO-FIELD INFLATION}

Planck data favor concave inflaton potentials, whereas convex ones occur more generically. A popular solution to this problem is through nonminimal coupling to gravity (see e.g., Ref. [22]), which in our framework would take the form

$$
\mathcal{L} \ni \frac{1}{2} m_{P}^{2} \zeta R\left(X^{2}+Y^{2}\right)
$$

where $R$ is the Ricci curvature. Transforming to the Einstein frame, the inflaton potential gets rescaled by $V(X, Y) \rightarrow V(X, Y) / \Omega^{4}$ where $\Omega^{2}=1+\zeta\left(X^{2}+Y^{2}\right)$, so that $V$ becomes concave at large field values.

However this simple device, while reconciling chaotic inflation potentials with CMB data, introduces a potentially strong coupling between the two fields, through the factor $\Omega^{-4}$. By numerical investigation we found that whenever $\zeta$ is large enough to resolve the tensions with CMB observations, it also causes the trajectories to align in the inflationary attractor solutions, $X \cong Y$, such that the effect of random initial differences $X_{i} \neq Y_{i}$ gets erased rather than enhanced during inflation, leading to nearly equal reheat temperatures in the two sectors. This is not compatible with the goal of the present work.

\section{II.1. Noncanonical models}

Another way of reconciling chaotic inflation potentials with CMB constraints is to use nonminimal kinetic

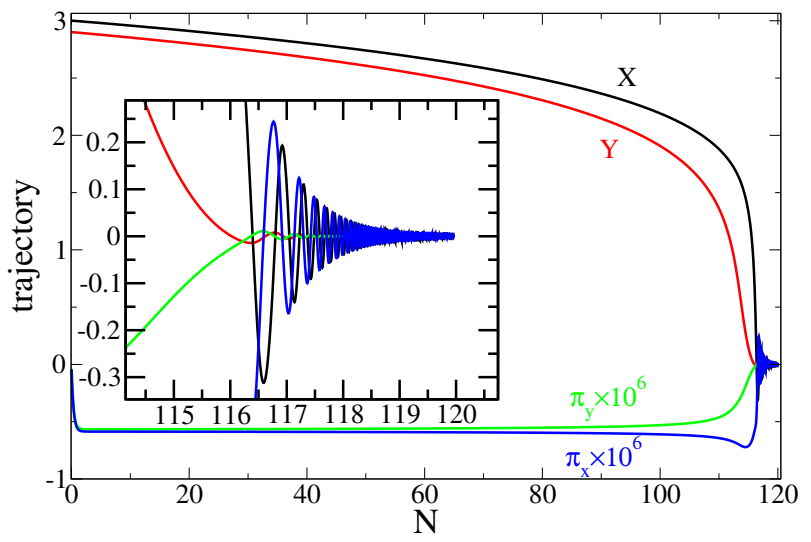

Figure 1. Illustrative trajectory for inflatons starting from nearly symmetric initial conditions in the model of Eq. (3), but achieving asymmetric reheating due to inflation ending earlier for the mirror inflaton $Y$. The inset highlights the end of inflation.

terms, for instance [23],

$$
\begin{aligned}
\mathcal{L} & =\frac{1}{2}\left(1+f \frac{X^{n}}{m_{P}^{n}}\right)(\partial X)^{2}+\frac{1}{2}\left(1+f \frac{Y^{n}}{m_{P}^{n}}\right)(\partial Y)^{2} \\
& +\frac{1}{2} m^{2}\left(X^{2}+Y^{2}\right) .
\end{aligned}
$$

For $X, Y \gg m_{P}$, the canonically normalized fields are $U \sim X^{1+n / 2}, W \sim Y^{1+n / 2}$, so that the potential becomes proportional to $\left(U^{4 /(n+2)}+W^{4 /(n+2)}\right)$. Here we will take the noncanonical fields to have a quadratic potential, as the simplest and most generic example.

The model of Eq. (3) essentially coincides with the power-law potential of Eq. (1) at large field values, with $p=4 /(2+n)$. The two scenarios only differ significantly near the end of inflation when the fields are close to the minimum of the potential, and most results from Ref. [9] also apply to our noncanonical scenario.

In the slow roll approximation, the equations of motion $3 H \dot{X}=-\partial V / \partial X$ and $3 H \dot{Y}=-\partial V / \partial Y$ for the potential of Eq. (1) can be integrated to show that 9]

$$
\begin{aligned}
& X / Y=\text { const }, p=2 ; \\
& X^{2-p}-Y^{2-p}=\text { const }, \quad p \neq 2 .
\end{aligned}
$$

Unlike the quadratic scenario, for $p \neq 2$ the inflaton trajectory is not a straight line in the $(X, Y)$ field space, unless the initial values $X_{i}$ and $Y_{i}$ are taken to be equal (or if one of them remains zero throughout inflation). If $p>2$, the two fields become synchronized before the end of inflation, since $X$ and $Y$ are both decreasing, and $X \approx Y$ when reheating takes place. This generally leads to both sectors reaching thermal equilibrium by the time of BBN, a possibility that is ruled out by the current bounds on $N_{\text {eff }}$.

The case $p<2$ is the most appealing for our purposes, since the fields are driven away from the symmetric tra- 
jectory $X=Y$. Then any initial randomly-occurring asymmetry is amplified by inflation, possibly leading to a difference of many orders of magnitude between the respective reheat temperatures. Moreover, potentials with $p \sim 2 / 3$ [12, 13] are generally consistent with Planck constraints on the tensor-to-scalar ratio $r$ and spectral index $n_{s}$ [10. However, in single-field inflation this agreement is marginal at best and is limited to a small range of powers $p$, as flatter potentials lead to to too-large predictions for $n_{s}$, and steeper ones give too-large $r$ [24]. We will show that in the two-field models the consistency is improved, for both the canonical and noncanonical implementations, and for a larger range of $p$ relative to single-field models.

In Fig. 1 we show an example of field trajectories for the noncanonical Lagrangian of Eq. (3) with an effective $p=2 / 3$ power-law potential $(n=4)$ and parameter values 2

$$
f=1, X_{i}=3, Y_{i}=2.9,
$$

where here and henceforth we work in Planck units with $m_{P}=1$, and assume vanishing initial velocities. Even though the initial values in this example differ only by $3 \%$, slow roll ends somewhat earlier in the $Y$ field, and its amplitude gets damped by a factor of $\sim 25$ relative to that of $X$, leading to a temperature ratio of $x \sim 0.2$, in agreement with the cosmological bound $x \lesssim 0.5$. Although we do not discuss the detailed mechanism of reheating in this work, the assumptions of unbroken mirror symmetry and complete conversion of the inflaton energies into radiation in the two respective sectors allows us to estimate the temperature ratio as

$$
x \equiv \frac{T^{\prime}}{T}=\left(\frac{\rho^{\prime}}{\rho}\right)^{1 / 4},
$$

by evaluating the energy densities at the end of inflation.

\section{II.2. Numerical solution}

To solve for the inflationary dynamics, we use the first order equations of motion for numerical integration, in terms of the canonical momenta $\pi_{x}=\partial \mathcal{L} / \partial \dot{X}=F(X) \dot{X}$, $\pi_{y}=\partial \mathcal{L} / \partial \dot{Y}=F(Y) \dot{Y}$, with $F(X) \equiv 1+f X^{n}$ for noncanonical models. It is convenient to take the number of $e$-foldings $N$ as the independent variable, defined through $d N=H d t$ in terms of the Hubble parameter $H$. Denot-

\footnotetext{
2 Throughout this work we fix the inflaton mass to $m=10^{-6}$ in the noncanonical model (no explicit mass term is present in the canonical version). Its value does not impact the inflationary dynamics during slow roll, but in a complete model it would set the scale of reheating, the details of which are not considered here.
}

ing $d / d N$ by primes, the system of equations is

$$
\begin{aligned}
X^{\prime} & =\frac{\pi_{x}}{F(X) H} \\
\pi_{x}^{\prime} & =-3 \pi_{x}+\frac{1}{H}\left(\frac{F^{\prime}(X)}{2}\left(\frac{\pi_{x}}{F(X)}\right)^{2}-m^{2} X\right) \\
Y^{\prime} & =\frac{\pi_{y}}{F(Y) H} \\
\pi_{y}^{\prime} & =-3 \pi_{y}+\frac{1}{H}\left(\frac{F^{\prime}(Y)}{2}\left(\frac{\pi_{y}}{F(Y)}\right)^{2}-m^{2} Y\right) \\
H & =\frac{1}{\sqrt{6}}\left(\frac{\pi_{x}^{2}}{F(X)}+\frac{\pi_{y}^{2}}{F(Y)}+m^{2}\left(X^{2}+Y^{2}\right)\right)^{1 / 2}
\end{aligned}
$$

Since we assume the two fields are decoupled from each other, each inflaton decays into its own matter sector during reheating. The resulting temperature ratio can therefore be estimated as in Eq. (6),

$$
x=\left(\frac{\frac{1}{2} F(Y)(\partial Y)^{2}+V(Y)}{\frac{1}{2} F(X)(\partial X)^{2}+V(X)}\right)^{1 / 4}
$$

evaluated at the end of inflation. Without loss of generality, we will assume $Y<X$ initially, so that $x<1$ and $X$ denotes the visible sector inflaton.

\section{II.3. Slow-roll parameters}

To compute the slow-roll parameters and inflationary observables it is convenient to work in the canonical basis, with fields denoted by $(U, W)$. Since the two kinetic terms in Eq. (3) are decoupled from each other, the Jacobian matrix $Z$ is diagonal,

$$
\left(\begin{array}{c}
\dot{X} \\
\dot{Y}
\end{array}\right)=\left(\begin{array}{cc}
F(X)^{-1 / 2} & 0 \\
0 & F(Y)^{-1 / 2}
\end{array}\right)\left(\begin{array}{c}
\dot{U} \\
\dot{W}
\end{array}\right) \equiv Z\left(\begin{array}{c}
\dot{U} \\
\dot{W}
\end{array}\right) .
$$

The slow-roll parameters computed in the canonical basis (indices $m, n$ ) are related to derivatives with respect to fields in the the original basis (indices $i, j$ ) by

$$
\begin{aligned}
\epsilon_{m} & =\frac{\left(Z_{i m} \partial_{i} V\right)^{2}}{2 V^{2}} \\
\eta_{m n} & =Z_{i m} Z_{j n} \frac{\partial_{i j} V}{V}+Z_{i m} \partial_{i} Z_{j n} \frac{\partial_{j} V}{V} .
\end{aligned}
$$

For numerical purposes, we modify these definitions by replacing $V \rightarrow \rho=3 H^{2}$ in the denominators. During inflation, this makes a negligible difference, whereas at the end of inflation while the inflaton is oscillating around its minimum, it avoids the artificial singularities that result from $V$ passing through zero.

The formalism for computing the spectral index $n_{s}$ of primordial adiabatic perturbations and the tensor-toscalar ratio $r$ in two-field models was developed in Refs. 
25, 26. One first introduces the adiabatic/entropy basis $(\sigma, s)$, defined by

$$
\begin{aligned}
d \sigma & =c_{\alpha} d U+s_{\alpha} d W, \\
d s & =-s_{\alpha} d U+c_{\alpha} d W,
\end{aligned}
$$

where $\alpha=\tan ^{-1}(\dot{W} / \dot{U})$ is the instantaneous slope of the field trajectory in the $U-W$ plane. The slow-roll parameters in the $(\sigma, s)$ basis are computed using [25]

$$
\begin{aligned}
\epsilon_{\sigma} & =\left(c_{\alpha} \partial_{U} V+s_{\alpha} \partial_{W} V\right)^{2} /\left(2 V^{2}\right) \\
\epsilon_{s} & \simeq 0 \\
\eta_{\sigma \sigma} & =c_{\alpha}^{2} \eta_{U U}+s_{\alpha}^{2} \eta_{W W} \\
\eta_{s s} & =s_{\alpha}^{2} \eta_{U U}+c_{\alpha}^{2} \eta_{W W} \\
\eta_{\sigma s} & =c_{\alpha} s_{\alpha}\left(\eta_{W W}-\eta_{U U}\right) .
\end{aligned}
$$

and the fact that $\eta_{U W}=0$ because $Z$ is diagonal $[c f$. Eq. (99)]. The derivatives of the potential in the $(U, W)$ basis are computed using the Jacobian matrix, $\partial_{m} V=$ $Z_{i m} \partial_{i} V$. Then to leading order in the slow-roll expansion, the scalar spectral index and tensor-to-scalar ratio are 26 .

$$
\begin{aligned}
n_{s}-1 & =-\left(6-4 c_{\Delta}^{2}\right) \epsilon_{\sigma}+2 s_{\Delta}^{2} \eta_{\sigma \sigma}+4 s_{\Delta} c_{\Delta} \eta_{\sigma s}+2 c_{\Delta}^{2} \eta_{s s} \\
r & =16 \epsilon_{\sigma}
\end{aligned}
$$

where $c_{\Delta}=-2 \mathcal{C} \eta_{\sigma s}, s_{\Delta}=+\sqrt{1-c_{\Delta}^{2}}, \mathcal{C}=2-\ln 2-\gamma \simeq$ 0.73 ( $\gamma$ is the Euler constant).

For the interpretation of the following results, it is useful to derive approximate expressions for $n_{s}$ and $r$ in terms of the background field amplitude and the canonical field ratio, defined respectively as

$$
\sigma \equiv\left(U^{2}+W^{2}\right)^{1 / 2}, \quad \xi \equiv \frac{W}{U} .
$$

This will help to elucidate how the two-field model improves over single-field inflation. In the large field limit, $\xi \cong \dot{W} / \dot{U}$, which is equivalent to approximating the instantaneous field velocity as being radial in the $U$-W plane. This implies that $\cos \alpha \approx U / \sigma$ and $\sin \alpha \approx W / \sigma$ in Eqs. 11 12). One can further approximate $\eta_{\sigma s} \ll 1$ so that $\cos ^{2} \Delta \sim 0$ in Eq. (13). Hence we obtain

$$
\begin{aligned}
n_{s}-1 & \approx-\frac{p(p+2)}{\sigma_{*}^{2}}-\frac{8 \mathcal{C} p^{2}(p-1)^{2}}{\sigma_{*}^{4}}\left(\frac{\xi_{*}^{p}-\xi_{*}^{2}}{\xi_{*}\left(1+\xi_{*}^{p}\right)}\right)^{2}, \\
r & \approx \frac{8 p^{2}}{\sigma_{*}^{2}},
\end{aligned}
$$

where $\sigma_{*}$ and $\xi_{*}$ are evaluated at horizon crossing, $N_{*} \approx$ $\sigma_{*}^{2} / 2 p$. The extra term in $n_{s}-1$, which is negative and arises from the term $4 s_{\Delta} c_{\Delta} \eta_{\sigma s}$ in Eq. (15)), explains why the spectral index comes into better agreement with Planck data than in single-field chaotic inflation. This term is explicitly related to the curvature of the potential and ensuing slow-roll trajectory, inspiring our CTHC (curved trajectory at horizon crossing) acronym. The ex- pression for $r$ is identical to that for single-field inflation 24.3

These are rough estimates, since Eq. (4) indicates $\dot{W} / \dot{U}=\xi^{p-1}$ during slow roll, in contrast to the approximation we have used. For small values of $\xi_{*} \lesssim 0.1$, the mirror inflaton is no longer slowly rolling and then higher order contributions to Eq. 13 become important. One can clearly see this breakdown in the approximations in the limit $\xi_{*} \rightarrow 0$, where the predictions for single-field inflation would be recovered in an exact expression. Nevertheless, Eq. (15) accurately describes the correlations between $\sigma_{*}, \xi_{*}$ and the cosmological observables when the two scalar fields are both slowly rolling during horizon exit, which is satisfied for $\xi_{*}$ not too much less than unity.

\section{II.4. Isocurvature fluctuations}

Two-field inflation models have the potential of generating isocurvature contributions to the power spectrum $\mathcal{P}_{S}(k)$, which are constrained by Planck. Thus, we compute the evolution of entropy perturbations to estimate their amplitude after inflation.

One can relate the adiabatic and entropy fluctuations at the end of inflation to their values at horizon crossing using a matrix of transfer functions [26] 28,

$$
\left(\begin{array}{l}
\mathcal{R} \\
\mathcal{S}
\end{array}\right)=\left(\begin{array}{ll}
T_{R R} & T_{R S} \\
T_{S R} & T_{S S}
\end{array}\right)\left(\begin{array}{l}
\mathcal{R}_{*} \\
\mathcal{S}_{*}
\end{array}\right),
$$

where the dimensionless adiabatic and isocurvature fluctuations are given by $\mathcal{R}=H \delta \sigma / \dot{\sigma}$ and $\mathcal{S}=H \delta s / \dot{\sigma}$ in the spatially flat gauge, and the star subscript indicates the time of horizon crossing. The matrix elements in the upper and lower rows are obtained by solving the perturbed equations of motion with initial conditions $\left(\mathcal{R}_{*}, \mathcal{S}_{*}\right)=(1,0)$ and $(0,1)$ respectively.

To compute the transfer functions of Eq. (16), it is more convenient to instead consider the evolution of the canonical fields' perturbations $\delta U, \delta W$ by making use of Eq. (11). Their equations of motion are [29]

$$
\begin{aligned}
\delta U^{\prime \prime}= & -C_{1} \delta U^{\prime}-3 \eta_{U U} \delta U+U^{\prime} \delta C \\
& +\left(U^{\prime 2}\right)^{\prime} \delta U+\left(U^{\prime} W^{\prime}\right)^{\prime} \delta W \\
\delta W^{\prime \prime}= & -C_{1} \delta W^{\prime}-3 \eta_{W W} \delta W+W^{\prime} \delta C \\
& +\left(W^{\prime 2}\right)^{\prime} \delta W+\left(U^{\prime} W^{\prime}\right)^{\prime} \delta U .
\end{aligned}
$$

Primes denote $d / d N, C_{1}=3+H^{\prime} / H$ and $\delta C=$ $C_{1}\left(U^{\prime} \delta U+W^{\prime} \delta W\right)$.

The amplitude of the isocurvature power spectrum is characterized by the scale-dependent primordial isocurvature fraction, $\beta(k)=\mathcal{P}_{S}(k) /\left[\mathcal{P}_{S}(k)+\mathcal{P}_{R}(k)\right]$, where

\footnotetext{
${ }^{3}$ In the numerical results, $r$ also depends weakly on $\xi_{*}$, but this dependence does not appear in Eq. 15 at the level of approximations we have used; to leading order $r$ is determined by $N_{*}$.
} 


\begin{tabular}{|c|c||c|c|c|c|c|c|c||c|c|c|c|}
\hline & \multicolumn{9}{|c||}{} & \multicolumn{9}{|c||}{ Noncanonical } & \multicolumn{4}{|c|}{ Canonical } \\
\hline$p$ & $x_{i}$ & $F(X)$ & $X_{i}$ & $Y_{i}$ & $f$ & $N_{\text {tot }}$ & $x_{f}$ & $\beta$ & $X_{i}$ & $Y_{i}$ & $N_{\text {tot }}$ & $x_{f}$ \\
\hline $2 / 3$ & 0.91 & $1+f X^{4}$ & 3.0 & 2.48 & 0.78 & 68.1 & $4.2 \times 10^{-3}$ & $5.8 \times 10^{-15}$ & 8.4 & 4.77 & 71.6 & $2.8 \times 10^{-4}$ \\
$1 / 2$ & 0.96 & $1+f X^{6}$ & 2.0 & 1.84 & 4.5 & 112.6 & $5.2 \times 10^{-7}$ & $1.6 \times 10^{-20}$ & 8.6 & 6.16 & 113.8 & $1.2 \times 10^{-3}$ \\
$1 / 3$ & 0.987 & $1+f X^{10}$ & 1.6 & 1.56 & 12 & 247.9 & $2.1 \times 10^{-8}$ & $1.8 \times 10^{-32}$ & 9.7 & 8.33 & 247.8 & $4.5 \times 10^{-3}$ \\
\hline
\end{tabular}

Table I. Parameters and initial values for three noncanonical benchmark models and their corresponding canonical power-law scenarios, $V \sim|X|^{p}+|Y|^{p}$, where $p=4 /(n+2)$. Parameters include the total number of $e$-foldings of inflation $N_{\text {tot }}$, the initial and final values of the temperature ratio $x$, and the amplitude of the isocurvature power spectrum on large scales $\beta$.

$\mathcal{P}_{R}(k)$ denotes the adiabatic power spectrum. On large scales, the leading contribution is [26]

$$
\beta \cong \frac{T_{S S}^{2}}{1+T_{S S}^{2}+T_{R S}^{2}} \lesssim \mathcal{O}\left(10^{-3}-10^{-2}\right)
$$

The experimental upper limit from Planck [10] depends on the assumptions of the fit and which datasets are used.

\section{BENCHMARK MODELS}

We start by considering three sets of benchmark parameters that illustrate the possibilities for phenomenologically successful inflation with a large reduction of temperature in the mirror sector. The results are presented for both the noncanonical models and their corresponding canonical versions in Table 1 and in Fig. 2 . Initial conditions were chosen such that the initial temperature ratios $x_{i}=\left(\rho_{i}^{\prime} / \rho_{i}\right)^{1 / 4} \sim 0.91-0.99$ were the same in both types of models. It can be seen in the figure that corresponding models lead to similar predictions for $n_{s}$ and $r$ (solid versus dashed lines).

The canonically normalized models with fractional power potentials are numerically challenging to evolve at late times while the fields are oscillating. During this regime, the fields undergo damped anharmonic motion and their energy density redshifts as 30 .

$$
\rho \sim a^{-6 p /(2+p)},
$$

where $a$ is the scale factor. As the amplitudes decrease, their frequency increases rapidly, impeding accurate numerical evolution. Hence for the canonical models we stop following the evolution of the mirror inflaton $Y$ once it reaches its minimum and instead extrapolate its energy density from the onset of oscillations to later times using Eq. 19.

The amplitude of the isocurvature power spectrum $\beta$ in noncanonical models was estimated by assuming a number of $e$-foldings at horizon crossing $N_{*}=55$. In every case, the predicted amplitude is much smaller than current bounds set by Planck $\rrbracket^{4}$ This can be understood from

\footnotetext{
${ }^{4}$ A similar conclusion applies for the model of Ref. [29], which was not recognized in that work.
}

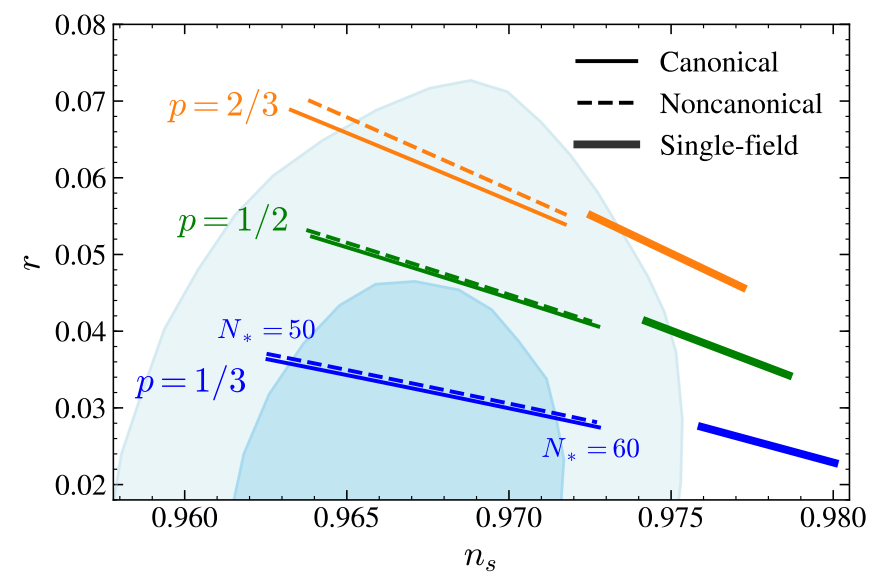

Figure 2. Spectral index and tensor-to-scalar ratio for the models of TableI for $N_{*} \in[50,60]$. The shaded regions show the 68 and $95 \%$ C.L. limits set by Planck. For noncanonical models, the effective power $p$ corresponds to $n=4 / p-2$ in Eq. (3). The thick lines to the right show the predictions in corresponding single-field chaotic inflation with the same fractional power-law potential.

the field trajectory of Fig. 1. since $Y$ reaches its minimum before the end of inflation, its perturbations are strongly diluted by the continuing exponential expansion of the universe. Although we could not determine $\beta$ very precisely in canonical power-law models, one expects that since the general dynamics are similar to noncanonical models, they should likewise lead to insignificant isocurvature perturbations.

All of the benchmark noncanonical models and their canonical counterparts lead to temperature ratios that are safely below the cosmological bound $x_{f}<0.5$, despite the initial ratio being close to 1 at the beginning of inflation. However, for $p<2 / 3$, noncanonical models lead to values of $x_{f}$ drastically lower than their canonical counterparts, $x \ll 10^{-3}$, a trend we will confirm below in a Monte Carlo analysis. In this limit the mirror sector would be almost unpopulated after reheating, an assumption that was made in e.g. Refs. [3, 31]. To our knowledge, the scenario presented here is the first one that can consistently predict such a cold mirror sector, consistent with CMB constraints, without requiring 
significant fine tuning in the initial conditions.

\section{III.1. Comparison with single-field power law inflation}

For a fixed initial temperature ratio $x_{i}$, the two types of models predict similar values for $n_{s}$ and $r$ if initial conditions are chosen such that the total duration of inflation $N_{\text {tot }}$ is approximately the same, as can be seen

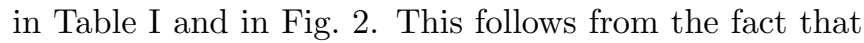
they coincide in the large field limit, and differ only in their behavior near the end of inflation. As predicted by Eq. 15, lower values of $p$ lead to lower predictions for the tensor-to-scalar ratio $r$, which tends to zero in the limit $p \rightarrow 0(n \rightarrow \infty)$. We can extrapolate our results to predict that a value of $r$ below 0.01 would require a power-law potential with $p \lesssim 0.15$, that is, $n \gtrsim 25$.

It is striking that the two-field inflationary scenarios are generally in much better agreement with Planck data than their single-field counterparts, represented by thick lines in Fig. 2. In single-field models, a flatter potential (lower $p$ ) decreases $r$ but also makes $n_{s}$ closer to 1 , such that $p=2 / 3$ and $p=1 / 2$ models are marginally consistent with Planck while $p=1 / 3$ is disfavored [24].

With two scalar fields rolling together, the Hubble parameter is increased by a factor of $\sqrt{2}$, effectively making each field roll more slowly at a given amplitude $5^{5}$ However this does not explain the better agreement of our models, since this effect preserves the dependences of $n_{s}$ and $r$ on $N_{*}$. In fact, if we set initial conditions $X_{i}=Y_{i}$, the failure of the single-field models persists, as can be seen by letting $\xi_{*}=1$ in Eq. (15).

Instead, the values of $n_{s}$ and $r$ in the two-field models depend on the difference in times for the end of inflation in the two sectors. As the mirror inflaton approaches its potential minimum, the field trajectory deviates from a straight line, and the extra curvature of the potential contributes to making the spectral index lower than what single-field inflation can achieve. This is the CTHC mechanism described by Eq. (15): since the field amplitude at horizon exit $\sigma_{*}$ is set by $N_{*}$, the main quantity that impacts $n_{s}$ is the canonical field ratio $\xi_{*}=W_{*} / U_{*}$. $\xi$ is strictly decreasing during inflation, and Eq. (15) is a monotonically increasing function of $\xi$ in the interval $[0,1]$. This implies that $\xi_{*}$ must fall within a precise window of values at $N_{*}$ to give optimal agreement with CMB observations [see Eq. 200]. Moreover, if the field ratio at the beginning of inflation $\xi_{i}$ is too small, that is, if the initial asymmetry between the fields is too big, the value of $n_{s}$ will be too small. In other words, the agreement between cosmological observations and the predictions of our model imposes a lower bound on the field ratio at the

\footnotetext{
5 'Assisted inflation' and 'N-flation' models are based on this idea 32 33. With $N$ independent scalar fields, $H$ is increased by $\sqrt{N}$, and the dynamics mimics that of a field at a higher scale.
}

beginning of inflation (assuming that $Y_{i}<X_{i}$ ). We will quantify this bound and the optimal range of $\xi_{*}$ at horizon crossing in the next section.

However, if $\xi_{*} \ll 1$ at horizon crossing, the approximate results of Eq. (15) are invalid and we instead recover the single-field limit ( $c f$ the thick lines in Fig. 2), since the mirror field makes a negligible contribution to inflation. Here the final temperature ratio can be arbitrarily small and isocurvature perturbations are essentially nonexistent, but the spectral index and the tensor-toscalar ratio are at best marginally consistent with $\mathrm{CMB}$ constraints. In the remainder of this work we will focus on the two-field inflationary models, where the mirror inflaton is still slowly rolling at horizon crossing.

Because the field ratio $\xi$ is closely related to the temperature ratio $x=T^{\prime} / T \approx(W / U)^{p / 4}$, Eq. 15 further implies a correlation between $n_{s}$ and $x_{f}$. This will allow us to identify ranges of $x_{f}$ that minimize the $\chi^{2}$ of observed versus predicted $n_{s}$ and $r$ values in the following section, that is, ranges of $x_{f}$ that are most consistent with CMB observations.

\section{MARKOV CHAIN MONTE CARLO}

To more completely explore the parameter space, we performed a Markov Chain Monte Carlo (MCMC) analysis for both the canonical and noncanonical models. The parameters that were allowed to vary were the initial field amplitudes and the kinetic parameter $f$ in Eq. (3), and we ran separate MCMCs for the values $p=1 / 3,1 / 2,2 / 3$, selecting models that fell within the Planck $95 \%$ confidence limits. Scatter plots showing correlations of the input parameter values from the resulting MCMC chains are presented in Fig. 3. They demonstrate the requirement of a small initial asymmetry $Y_{i} \cong X_{i}$, and an inverse correlation between $f$ and $X_{i}$ that is necessary for achieving a long enough period of inflation. Fig. 3 shows that smaller initial field values are required in the noncanonical models, but in terms of the canonically normalized fields, e.g., $U=\int F(X)^{1 / 2} d X$, the initial values are of the same order $(\gtrsim 10)$ for both kinds of models.

\section{IV.1. Inflationary observables}

As explained above, canonical and noncanonical models lead to very similar predictions for $n_{s}$ and $r$, differing primarily in their predicted final temperature ratios. Fig. 4 shows these predictions, as well as the correlation between the total duration of inflation $N_{\text {tot }}$ and the initial temperature asymmetry $x_{i}$, for both kinds of models. There is no apparent distinction between the two kinds of models in these plots. For the value of $N_{*}$ at which $n_{s}$ and $r$ were evaluated in each model, we chose a random number in the interval $[50,60]$, to reflect the uncertainty in the overall scale of inflation and reheat temperatures. 

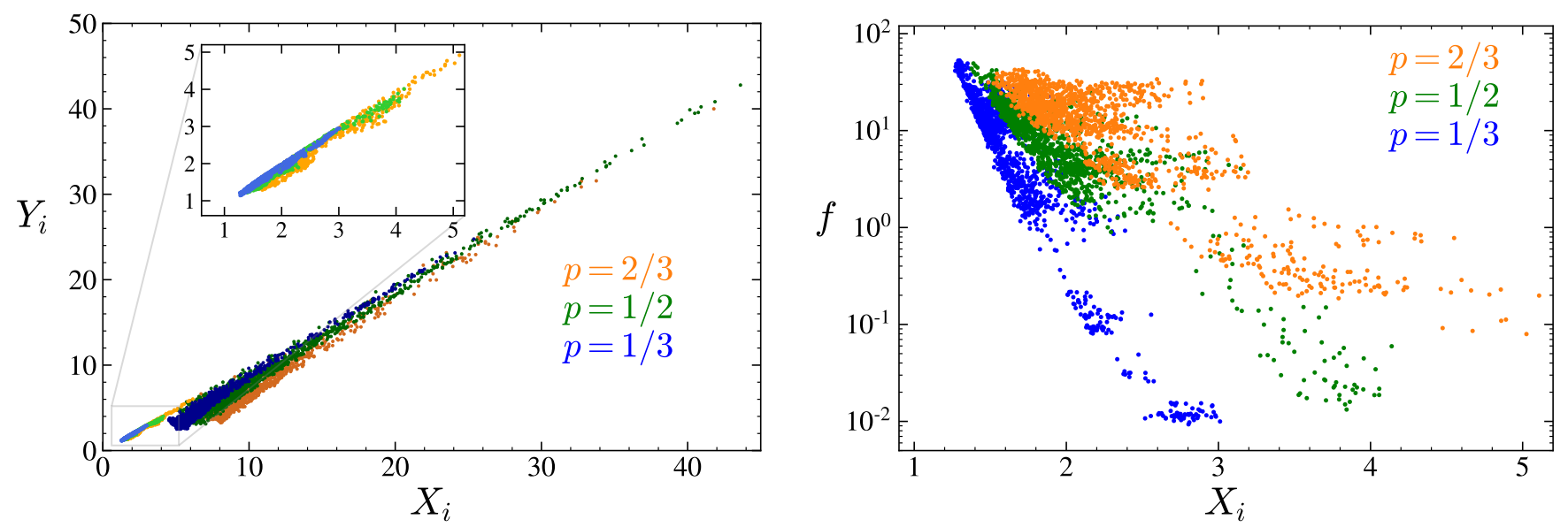

Figure 3. Left: correlation of initial conditions in the MCMC chains. Dark (light) colors correspond to canonical (noncanonical) models. The inset shows the noncanonical models only. Right: correlation of $f$ versus $X_{i}$ for noncanonical models.
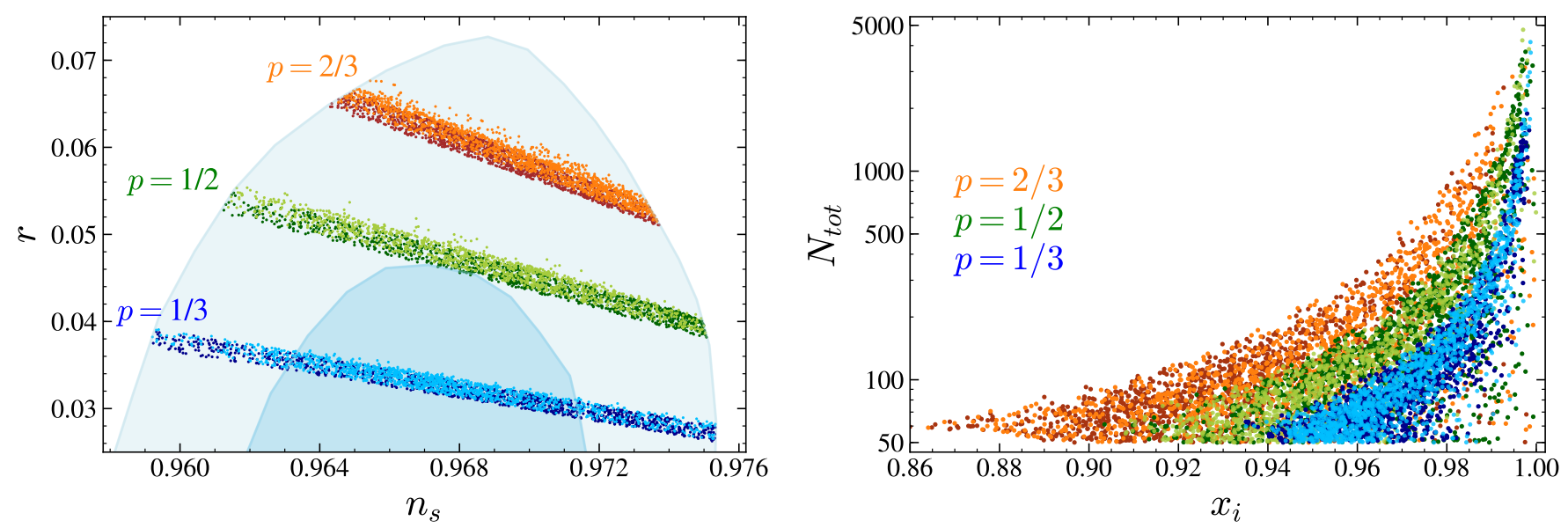

Figure 4. Scatter plots from the Markov Chain Monte Carlo (MCMC) analysis. Left: correlation between $n_{s}$ and $r$. Right: correlation of the initial temperature asymmetry $x_{i}=T_{i}^{\prime} / T_{i}$ with the total number of $e$-foldings during inflation $N_{t o t}$. Dark (light) colors correspond to canonical (noncanonical) models, but there is no significant distinction between the two types of scenarios within these plots. All models are within the $95 \%$ C.L. limits of Planck.

We observe a significant variation for the value of the spectral index (and, to a lesser extent, for the tensorto-scalar ratio) within each class of models depending on the parameters and initial conditions. This is the result of the dependence of $n_{s}$ on both $N_{*}$ and the field ratio $\xi_{*}$ at horizon crossing described by Eq. 15), and it demonstrates the versatility of the two-field models in contrast to single-field inflation.

The right panel of Fig. 4 can be understood in terms of the CTHC effect described previously: if the fields are close to each other at the beginning of inflation $\left(x_{i}=\xi_{i}^{p / 4} \approx 1\right)$, then inflation must last longer in order for the trajectory to bend enough to reach the ratio $\xi_{*}=\left(Y_{*} / X_{*}\right)^{2 / p}$ at horizon crossing that corresponds to optimal agreement with CMB observations. One can achieve a longer period of slow-roll either by increasing the initial values of the fields or the nonmiminal kinetic parameter $f$. Numerically, we find that

$$
0.4 \lesssim \xi_{*} \lesssim 0.7
$$

is required at horizon exit for $n_{s}$ to fall within Planck's $68 \%$ confidence limit, $n_{s}=0.9649 \pm 0.0042$ [10], although the precise interval depends mildly on $p$.

It is noteworthy that for a given value of $p$, there exists a minimum value of $x_{i}$, below which no models were retained in our MCMC chains. This can be understood in terms of the optimal range 20 for $\xi_{*} \cong x_{*}^{p / 4}$. If $x_{i}$ falls below the corresponding lower bound of Eq. (20), $\xi_{i} \lesssim 0.4^{p / 4}$, then $x(t)$ continues to decrease during inflation, resulting in a value of $n_{s}$ that is too small no matter how long inflation lasts, in conflict with Planck data. These minimum values are rather close to $1: x_{i} \gtrsim 0.86$, 0.92 and 0.94 for $p=2 / 3,1 / 2$ and $1 / 3$, respectively. Therefore, the flatter the potential is, the closer the en- 

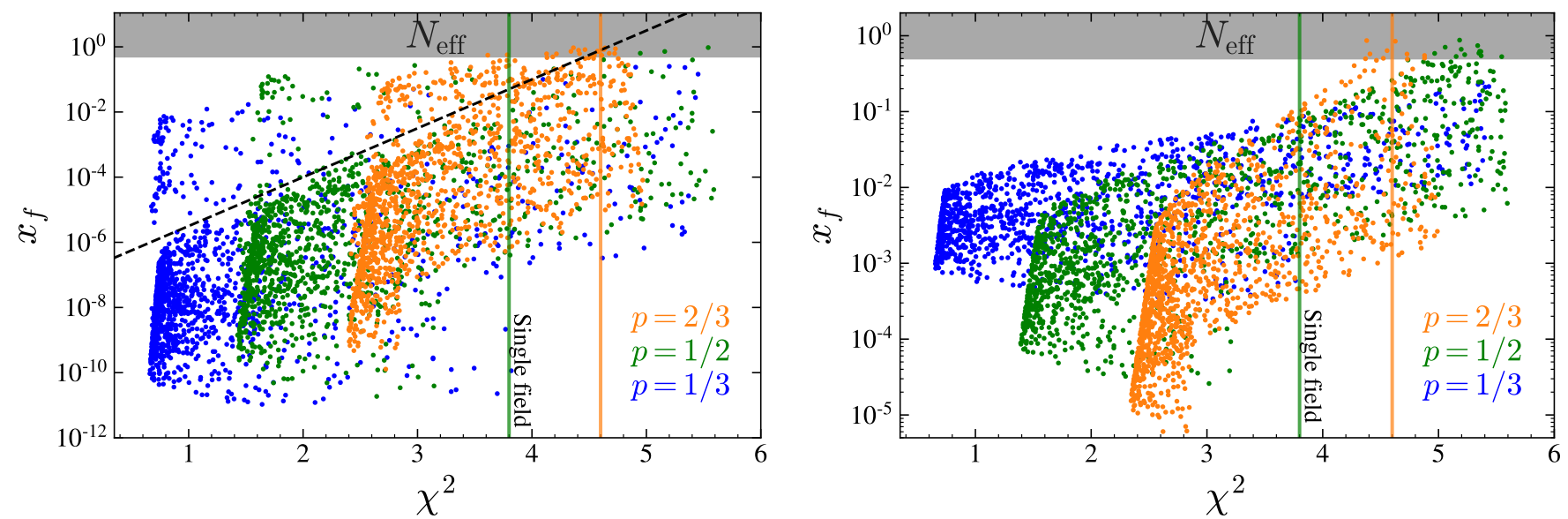

Figure 5. Correlation between the final temperature ratio $x_{f}$ and $\chi^{2}$ for noncanonical (left) and canonical (right) models from the MCMC analysis, that respect the 95\% C.L. limits of Planck. The grey shading at the top indicates the excluded region $x_{f}>0.5$. For comparison, the vertical lines show the minimal value of $\chi^{2}$ for single-field $p=1 / 2$ (green) and $p=2 / 3$ (orange) inflation models. Data points above the dashed line in the left panel correspond to models where the oscillations of $Y$ are overdamped, as explained in the text.

ergy densities must be initially. Whether this could be considered as a mild fine tuning of initial conditions cannot be quantified in the absence of a complete theory for the probability distribution of the field amplitudes at the beginning of inflation. It is surprising and somewhat ironic that our search for a large temperature asymmetry reveals the requirement of a moderate level of symmetry in the initial conditions, to achieve agreement with current cosmological observations.

\section{IV.2. Temperature ratio}

It is striking that our mechanism can produce final temperature asymmetries as great as $x_{f} \sim 10^{-10}$ (for the noncanonical models), and it rarely gives values $x_{f} \gtrsim 0.5$ that are in the cosmologically excluded region. This is illustrated in Fig. 5, which shows the correlation between $x_{f}$ and the value of $\chi^{2}$ with respect to $n_{s}$ and $r$. The most important feature is the difference of scale between the vertical axes of the two panels: the noncanonical models can lead to dramatically lower $x_{f}$ than the canonical ones.

The pertinent difference between the two kinds of models arises from the equation of state of the fields near the minimum of the potential. Since we assume the canonical potential retains its fractional power-law shape in the oscillatory regime, the energy density of the mirror inflaton $Y$ redshifts as in Eq. 19p once it starts oscillating. One can see that higher values of $p$ make $Y$ decay faster, which is why $p=2 / 3$ canonical models may lead to a temperature ratio as low as $x_{f} \sim 10^{-5}$ while $p=1 / 3$ models are limited to $x_{f} \sim 10^{-3}$ at best. These conclusions could change if the shape of the potential was different near its minimum ${ }^{6}$ In contrast, the noncanonical models have a quadratic potential at small field values, leading to same equation of state as cold dark matter after the end of inflation: $\rho \sim a^{-3}$. Hence in these models the mirror inflaton generally decays much faster than in canonical models, explaining why the final temperature ratio can be as low as $\sim 10^{-10}$.

Exceptionally, the left panel of Fig. 5 reveals some noncanonical models that lead to small values of $\chi^{2}$, yet with much larger temperature ratios. These points are separated from the rest by a dashed line to highlight the distinct correlations. They correspond to models where the nonminimal kinetic parameter $f$ is small $(\lesssim 1, c f$. the lower part in the second panel of Fig. 3). Such values of $f$ generally require larger initial amplitudes $X_{i}$ and $Y_{i}$ to maintain the requirement 20$)$ on $\xi_{*}$. This in turn makes the Hubble parameter larger, increasing the impact of damping on the oscillations of $Y$ in its oscillating phase.

Elaborating on this point, by neglecting the nonminimal kinetic term after $Y$ leaves the slow rolling regime, the equation of motion of the mirror inflaton is

$$
Y^{\prime \prime}+3 Y^{\prime}+\frac{m^{2}}{H^{2}} Y \approx 0,
$$

which describes a damped harmonic oscillator. When $H / m>2 / 3$, the oscillations of $Y$ are overdamped, making its amplitude decay very slowly. By contrast, if

\footnotetext{
${ }^{6}$ For instance, axion monodromy models 13 typically have a potential of the form $V(X) \sim\left(X^{2}+\epsilon^{2}\right)^{p / 2}$, which looks like a power-law when $X \gg \epsilon$ while being approximately quadratic near the minimum, when $X \ll \epsilon$. This scenario would be very similar to our noncanonical model since they would both yield the same equation of state at small field amplitude.
} 
$H / m<2 / 3$ when $Y$ enters its oscillating phase it is underdamped and it decays exponentially. Models above the dashed line in the right panel of Fig. 5 all correspond to the overdamped case, which is why the final temperature ratio is many orders of magnitude above the low-amplitude cases. This behavior occurs only in the noncanonical models because there both $f$ and the initial conditions $X_{i}, Y_{i}$ impact the duration of inflation; in canonical scenarios only the latter play a role.

\section{IV.3. Predictions for $x_{f}$ and isocurvature}

Although the final temperature ratios $x_{f}$ span many orders of magnitude in the MCMC results, if future CMB measurements converge on values of $n_{s}$ and $r$ near the Planck best-fit values, this range can become narrower. This is due to the correlation between the spectral index and the field ratio given by Eq. (15), which implies a correlation between $x_{f}$ and $n_{s}$. Hence only certain values of $x_{f}$ are predicted by models that yield $n_{s}=0.9649 \pm$ 0.0042. The right panel of Fig. 5, which refers to the canonical models, predicts e.g. $\sim 10^{-3}-10^{-2}$ for $p=$ $1 / 3$. Wider intervals are allowed for the underdamped noncanonical models (below the dashed line of the left panel), e.g., the data points cluster near $\chi^{2} \approx 0.6$ for $x_{f} \sim 10^{-10}-10^{-6}$ for $p=1 / 3$, while the possibility of overdamped models extends the most likely range of $x_{f}$ to up to $\sim 10^{-2}$.

Fig. 5 shows, by the vertical lines, the minimal values of $\chi^{2}$ obtainable in single-field $p=2 / 3$ and $p=1 / 2$ inflationary model. Neither model gives a good fit to the data, and the $p=1 / 3$ case is disfavored at $>95 \%$ C.L. (see Fig. 22), so its corresponding horizontal line falls outside the plotted region. The single-field models also describe two-field inflation in the regime where $Y \ll X$ at horizon crossing, so that $x_{f}$ can be arbitrarily small in those scenarios. However, most points from our MCMC analysis give better fits than the single-field models.

Similarly to the benchmark models of section [II] we computed the amplitude of the isocurvature power spectrum $\beta$ for noncanonical models in the MCMC chains. Fig. 6 shows the resulting correlation between $\beta$ and $x_{f}$. As anticipated, the $\beta$ is too small to be observable in most cases, with only a handful of $p=2 / 3$ models approaching the sensitivity of Planck. We empirically observe a scaling relation $\beta \sim x_{f}^{4}$. Even though models with observable isocurvature are rare, these examples are interesting because they suggest that a detection of isocurvature could be correlated with an observable deviation in $N_{\text {eff }}$.

\section{CONCLUSION}

In this work we have revisited a mechanism for generating a cooler mirror sector from inflation, due to random initial conditions, while maintaning exact mirror symmetry at the Lagrangian level. It was shown that two-field

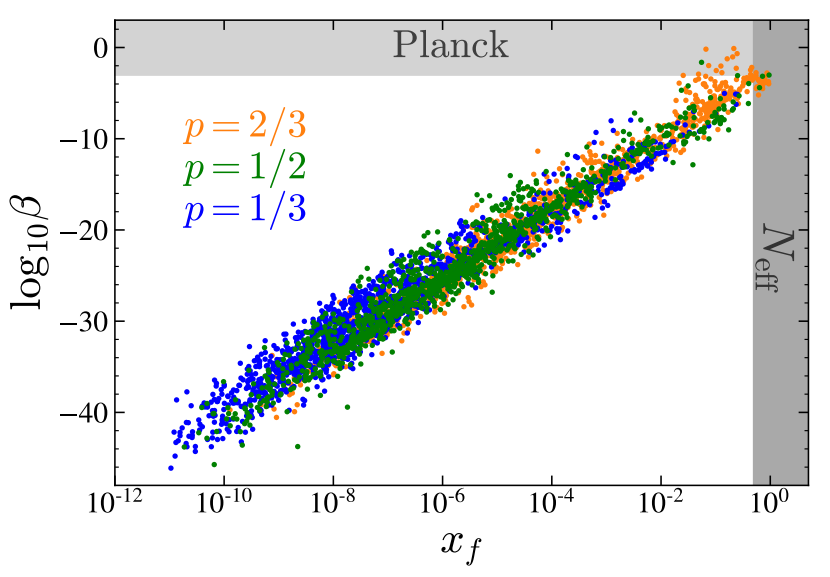

Figure 6. Correlation between the amplitude of the isocurvature spectrum $\beta$ on large scales and the final temperature ratio $x_{f}$ in the noncanonical models. The grey bands are the excluded region $x_{f}>0.5$ and the limit on the Planck sensitivity $\beta>10^{-3}$.

inflation models with a fractional power-law potential can efficiently amplify a small asymmetry between the visible sector and its mirror counterpart, leading to a temperature ratio as low as $x_{f} \sim 10^{-10}$ at the end of inflation. Models with a nonminimal kinetic term generally lead to values of $x_{f}$ that are much lower than the corresponding canonically normalized scenarios, due to the difference in the equation of state of the fields when they are oscillating around the minimum of the potential.

While both kinds of models can be in much better agreement with Planck data than single-field inflation, somewhat surprisingly this requires the initial temperature asymmetry between the two sectors to be relatively small, $x_{i} \gtrsim 0.86$. The value of the tensor-to-scalar ratio $r$ depends on the effective power $p$ of the potential, lying in the range $\sim 0.03-0.07$ for $p$ between $1 / 3$ and $2 / 3$, which could be observed in upcoming CMB experiments 34.

A key assumption is that the two sectors are decoupled or very weakly interacting with each other. For example, a $\lambda_{H H^{\prime}}|H|^{2}\left|H^{\prime}\right|^{2}$ coupling mixing the Higgs bosons of the two sectors must have $\lambda_{H H^{\prime}} \lesssim 10^{-8}$ to avoid equilibration of the temperatures after reheating. 7 This is technically natural since $\lambda_{H H^{\prime}}$ is only multiplicatively renormalized.

On the other hand we have also assumed the possible interaction $\lambda_{X Y} X^{2} Y^{2}$ to be absent. One might expect that its presence could synchronize the two fields during inflation and make $X \approx Y$ at the onset of reheating. In a preliminary investigation we find the opposite behavior: nonvanishing $\lambda_{X Y}$ instead tends to enhance the final temperature asymmetry, naively estimated as we have done throughout this work. However whether this would

\footnotetext{
7 By demanding the scattering rate $\Gamma \sim \lambda_{H H^{\prime}}^{2} T<H \sim T^{2} / M_{p}$ down to the weak scale $T \sim m_{H}$.
} 
be a good estimate in the present case is questionable, because of the possibility of $Y$ particle productions via parametric resonance or $X X \rightarrow Y Y$ scattering during reheating. This question is beyond the scope of the present work, but could be interesting for future study.
Acknowledgment. We thank Eva Silverstein for helpful correspondence. This work was supported by NSERC (Natural Sciences and Engineering Research Council, Canada).
[1] I. Y. Kobzarev, L. B. Okun, I. Y. Pomeranchuk, On the possibility of experimental observation of mirror particles, Sov. J. Nucl. Phys. 3 (6) (1966) 837-841.

[2] L. B. Okun, Mirror particles and mirror matter: 50 years of speculations and search, Phys. Usp. 50 (2007) 380-389. arXiv:hep-ph/0606202, doi:10.1070/ PU2007v050n04ABEH006227.

[3] R. Foot, Mirror dark matter: Cosmology, galaxy structure and direct detection, Int. J. Mod. Phys. A 29 (2014) 1430013. arXiv:1401.3965, doi:10.1142/ S0217751X14300130.

[4] Z. Berezhiani, D. Comelli, F. L. Villante, The Early mirror universe: Inflation, baryogenesis, nucleosynthesis and dark matter, Phys. Lett. B 503 (2001) 362-375. arXiv:hep-ph/0008105, doi:10.1016/S0370-2693(01) 00217-9

[5] J.-S. Roux, J. M. Cline, Constraining galactic structures of mirror dark matter (2020). arXiv:2001.11504.

[6] D. Curtin, S. Gryba, Twin Higgs Portal Dark Matter (1 2021). arXiv:2101.11019.

[7] N. Craig, I. Garcia Garcia, G. Koszegi, A. McCune, P not PQ (12 2020). arXiv:2012.13416

[8] Z. Berezhiani, A. Dolgov, R. Mohapatra, Asymmetric inflationary reheating and the nature of mirror universe, Phys. Lett. B 375 (1996) 26-36. arXiv:hep-ph/9511221, doi:10.1016/0370-2693(96)00219-5

[9] V. S. Berezinsky, A. Vilenkin, Ultrahigh-energy neutrinos from hidden sector topological defects, Phys. Rev. D62 (2000) 083512. arXiv:hep-ph/9908257, doi:10.1103/ PhysRevD.62.083512

[10] Y. Akrami, et al., Planck 2018 results. X. Constraints on inflation (2018). arXiv:1807.06211.

[11] E. W. Kolb, D. Seckel, M. S. Turner, The shadow world of superstring theories, Nature 314 (6010) (1985) 415-419. doi: $10.1038 / 314415 \mathrm{a} 0$.

[12] E. Silverstein, A. Westphal, Monodromy in the CMB: Gravity Waves and String Inflation, Phys. Rev. D 78 (2008) 106003. arXiv:0803.3085 doi:10.1103/ PhysRevD.78.106003

[13] L. McAllister, E. Silverstein, A. Westphal, Gravity Waves and Linear Inflation from Axion Monodromy, Phys. Rev. D 82 (2010) 046003. arXiv:0808.0706, doi:10.1103/ PhysRevD.82.046003

[14] X. Dong, B. Horn, E. Silverstein, A. Westphal, Simple exercises to flatten your potential, Phys. Rev. D 84 (2011) 026011. arXiv:1011.4521 doi:10.1103/PhysRevD.84. 026011 .

[15] G. Gur-Ari, Brane Inflation and Moduli Stabilization on Twisted Tori, JHEP 01 (2014) 179. arXiv:1310.6787, doi:10.1007/JHEP01(2014) 179

[16] L. McAllister, E. Silverstein, A. Westphal, T. Wrase, The Powers of Monodromy, JHEP 09 (2014) 123. arXiv: 1405.3652, doi:10.1007/JHEP09(2014) 123

[17] F. Marchesano, G. Shiu, A. M. Uranga, F-term Axion
Monodromy Inflation, JHEP 09 (2014) 184. arXiv:1404. 3040, doi:10.1007/JHEP09(2014) 184

[18] S. Bielleman, L. E. Ibanez, F. G. Pedro, I. Valenzuela, C. Wieck, The DBI Action, Higher-derivative Supergravity, and Flattening Inflaton Potentials, JHEP 05 (2016) 095. arXiv:1602.00699, doi:10.1007/JHEP05(2016) 095

[19] A. Landete, F. Marchesano, G. Shiu, G. Zoccarato, Flux Flattening in Axion Monodromy Inflation, JHEP 06 (2017) 071. arXiv:1703.09729, doi:10.1007/ JHEP06 (2017) 071

[20] X. Gao, T. Li, P. Shukla, Fractional chaotic inflation in the lights of PLANCK and BICEP2, Phys. Lett. B 738 (2014) 412-417. arXiv:1404.5230, doi:10.1016/j. physletb.2014.10.007.

[21] D. Wenren, Tilt and Tensor-to-Scalar Ratio in Multifield Monodromy Inflation (5 2014). arXiv:1405.1411

[22] A. Linde, M. Noorbala, A. Westphal, Observational consequences of chaotic inflation with nonminimal coupling to gravity, JCAP 03 (2011) 013. arXiv:1101.2652 doi:10.1088/1475-7516/2011/03/013

[23] H. M. Lee, Chaotic inflation and unitarity problem, Eur. Phys. J. C74 (8) (2014) 3022. arXiv:1403.5602, doi: 10.1140/epjc/s10052-014-3022-0.

[24] P. A. R. Ade, et al., Planck 2013 results. XXII. Constraints on inflation, Astron. Astrophys. 571 (2014) A22. arXiv:1303.5082, doi:10.1051/0004-6361/201321569

[25] C. Gordon, D. Wands, B. A. Bassett, R. Maartens, Adiabatic and entropy perturbations from inflation, Phys. Rev. D 63 (2000) 023506. arXiv:astro-ph/0009131, doi:10.1103/PhysRevD.63.023506

[26] C. T. Byrnes, D. Wands, Curvature and isocurvature perturbations from two-field inflation in a slow-roll expansion, Phys. Rev. D 74 (2006) 043529. arXiv:astro-ph/ 0605679, doi:10.1103/PhysRevD.74.043529

[27] L. Amendola, C. Gordon, D. Wands, M. Sasaki, Correlated perturbations from inflation and the cosmic microwave background, Phys. Rev. Lett. 88 (2002) 211302. arXiv:astro-ph/0107089, doi:10.1103/PhysRevLett. 88.211302

[28] D. Wands, Multiple field inflation, Lect. Notes Phys. 738 (2008) 275-304. arXiv:astro-ph/0702187, doi: 10.1007/978-3-540-74353-8_8.

[29] J. M. Cline, M. Puel, T. Toma, Affleck-Dine inflation, Phys. Rev. D 101 (4) (2020) 043014. arXiv:1909.12300, doi:10.1103/PhysRevD.101.043014.

[30] M. S. Turner, Coherent Scalar Field Oscillations in an Expanding Universe, Phys. Rev. D 28 (1983) 1243. doi: 10.1103/PhysRevD.28.1243

[31] R. Foot, Mirror dark matter cosmology - predictions for $N_{e f f}[C M B]$ and $N_{e f f}[B B N]$, Phys. Lett. B 711 (2012) 238-243. arXiv:1111.6366, doi:10.1016/j.physletb. 2012.04.023.

[32] A. R. Liddle, A. Mazumdar, F. E. Schunck, Assisted in- 
flation, Phys. Rev. D 58 (1998) 061301. arXiv:astro-ph/ 9804177, doi:10.1103/PhysRevD.58.061301

[33] S. Dimopoulos, S. Kachru, J. McGreevy, J. G. Wacker, N-flation, JCAP 08 (2008) 003. arXiv: hep-th/0507205 doi:10.1088/1475-7516/2008/08/003

[34] M. Hazumi, et al., LiteBIRD: A Satellite for the Studies of B-Mode Polarization and Inflation from Cosmic Background Radiation Detection, J. Low Temp. Phys. 194 (56) (2019) 443-452. doi:10.1007/s10909-019-02150-5 